Freixa Niella, M.; Llanes Ordóñez, J. y Venceslao Pueyo, M. (2018). El abandono en el recorrido formativo del estudiante de ADE de la Universidad de Barcelona. Revista de Investigación Educativa, 36(1), 185-202.

DOI: http://dx.doi.org/10.6018/rie.36.1.278971

\title{
El abandono en el recorrido formativo del estudiante de ADE de la Universidad de Barcelona
}

\author{
Abandonment in the Student's educational itinerary \\ of ADE of the University of Barcelona
}

\author{
Montserrat Freixa Niella, Juan Llanes Ordóñez y Marta Venceslao Pueyo \\ Departamento de Métodos de Investigación y Diagnóstico en Educación. Facultad de Educación. \\ Universidad de Barcelona (España)
}

\begin{abstract}
Resumen
El presente artículo corresponde a un análisis del abandono de la carrera de ADE en primer, segundo y tercer curso; las diferentes causas que hacen que el alumnado abandone y los motivos. La metodología es de carácter cuantitativo descriptivo, utilizando un cuestionario como instrumento de recogida de información. Se presentan, en primer lugar, el análisis cuantitativo de los motivos de abandono y, en segundo, el análisis del discurso de los estudiantes. La investigación demuestra que el abandono universitario no puede interpretarse únicamente como abandono del sistema educativo puesto que un número importante de estudiantes reingresa en otros grados universitarios o ciclos formativos. Sitúa, a su vez, claras diferencias en las causas de abandono para aquellos estudiantes que simultanean trabajo y estudios y los que sólo estudian. Para estos últimos, la desmotivación y las expectativas no cumplidas en relación a la carrera sería una causa fundamental. Encontramos otros motivos de abandono preeminentes en las dificultades de compaginación entre trabajo y estudios; el fracaso académico y la construcción de una identidad profesional débil.
\end{abstract}

Palabras clave: abandono; universidad; educación superior; orientación.

Correspondencia: Montserrat Freixa Niella, mfreixa@ub.edu, Passeig de la Vall d'hebron 171 (edifici llevant), C.P. 08035 (Barcelona).

El artículo es resultado de una investigación procedente de un proyecto MECD, I+D, EDU 2009-10351, EDU 2012-31563s 


\begin{abstract}
This article focuses on the analysis of the abandonment of ADE's career in the first, second and third years. It aims to analyze the moment of abandonment throughout the study period, to identify the different causes that make/cause students abandon; it also describes the abandonment reasons over the three years. The methodology is descriptive in quantitative terms, using a questionnaire as a tool for collecting information. We present, firstly, the quantitative analysis of the reasons for abandonment and, secondly, the analysis of the students' discourse. Research shows that university dropout cannot be interpreted solely as abandonment of the education system since a significant number of students re-enter other university degrees or training cycles. It establishes, in turn, clear differences in the causes of abandonment for those students who simultaneously work and study and those who only study. For the latter, demotivation and unfulfilled expectations in relation to the career would be a fundamental cause. We find other reasons for abandonment preeminent in the difficulties of reconciliation between work and studies; academic failure, lack of motivation and the construction of a weak professional identity.

Keywords: dropping out; university; higher education; guidance.
\end{abstract}

\title{
Introducción
}

El fenómeno del abandono en la educación superior ha devenido objeto de estudio de las ciencias sociales durante las últimas décadas. La preocupación social e institucional ha espoleado tanto la elaboración de modelos y teorías explicativas, como el debate conceptual y operativo sobre la precisión en la terminología empleada. En el caso del Estado Español, la prevalencia del problema del abandono universitario ha sido analizada profusamente por autores como Arce, Crespo y Miguez-Álvarez (2015), Bethencourt, Cabrera, Hernández, Álvarez y González (2008), Escandell, Marrero y Rubio (1999) o Torrado (2012), entre otros.

Este interés ha permitido una mayor comprensión de la complejidad de las circunstancias y características de las personas que deciden abandonar o continúan sus estudios y plantear las políticas de retención a partir de medidas que abarcan desde programas de orientación, hasta políticas de becas y ayudas institucionales (Berlanga, Figuera \& Pons-Fanals, 2013).

El abandono nos remite a una variedad de situaciones identificadas en el proceso educativo con un denominador común: detención o interrupción de estudios iniciados antes de finalizarlos (Cabrera, Burkum, La Nasa \& Bibo, 2012). Conviene diferenciar, en este sentido, el abandono voluntario del forzoso o no voluntario, y deserción tardía, de primera deserción. Las tasas más elevadas de abandono son de carácter voluntario y se producen durante el primer curso. Los datos del Ministerio de Educación, Cultura y Deporte (MECD, 2015) sitúan que uno de cada cinco estudiantes abandona en el primer curso. Durante los dos primeros años se sitúa en el $20.7 \%$ y la de cambio de estudio en el $10 \%$.

En el marco de estas consideraciones, distintas investigaciones (Beaupère, Chalumeau, Gury \& Hugree, 2007; Figuera, Torrado, Dorio \& Freixa, 2015) sitúan, por un lado, que la decisión de abandonar ocupa un tiempo concreto en las trayectorias académicas del estudiante y, por otro, que existen momentos de especial sensibilidad frente a la decisión 
de abandono como el inicio del curso y el momento posterior a los primeros resultados. Estudios como Krause y Coates (2008) o Yorke y Longden (2008) destacan la importancia de las primeras experiencias universitarias para explicar la adaptación a la universidad a largo plazo. Sin embargo, existen pocas investigaciones que se centren en el abandono más allá del primer curso. El trabajo de Nora y Crips (2012) y la tesis doctoral de Torrado (2012) confirman perfiles diferenciales en las trayectorias de abandono cuando se incluye el tiempo o el curso en el que acaece el abandono.

La literatura especializada señala que el abandono universitario es un fenómeno de naturaleza multicausal en el que intervienen situaciones de diversa índole como la elección de la carrera, el rendimiento o la tipología de estudiantes. Todas ellas se inscriben en un conjunto de factores personales e institucionales significativos para el abandono (Bethencourt et al. 2008). Algunos estudios, como el de Cabrera et al. (2012), subrayan que las variables del alumnado son más influyentes que las del contexto. Y dentro de aquellas, las más determinantes serían las características psicológicas y las estrategias de estudio. En esa misma línea, Escandell et al. (1999) sostienen que los factores de carácter psicoeducativo son los que mayor peso explicativo tienen en el abandono.

La elección de la carrera, constituye un factor relevante para el abandono (Corominas, 2001). El conocimiento sobre los estudios a cursar y los motivos de la elección conforman unas expectativas que serán confrontadas permanentemente en el transcurso de la carrera. Navarro y Soler (2014) distinguen tres tipos de motivaciones para la elección de la carrera: la expresiva (motivación intrínseca: "es la carrera que me gusta"), la instrumental (motivación extrínseca: aspectos económicos) y la profesional (proyecto de vida: profesión que desea ejercerse). Nuestra investigación sitúa para el grado de Administración y Dirección de Empresas (ADE, en adelante), un alto índice de motivación instrumental entre los estudiantes y una motivación expresiva moderada. No obstante, el conocimiento que el alumnado tiene sobre los estudios elegidos es, por lo general, escaso (Álvarez, López \& Pérez-Jorge, 2015), cuestión que invita a continuar indagando en los factores de elección y sus consecuencias para articular medidas de orientación eficaces (Álvarez, 2015; Romero \& Figuera, 2016).

El rendimiento académico es otro de los factores principales de abandono (Figuera \& Torrado, 2013; Gairín, Figuera \& Triadó, 2010; Torrado, 2012;). La obtención de resultados académicos inferiores a los esperados produce una desmotivación que puede desembocar en abandono. En esta línea, también cabría considerar los estudios que vinculan el rendimiento académico con las estrategias de estudios y los planes formativos de los grados (Braxton, Hirschy \& McClendon, 2004; Mingorance \& Calvo, 2012; Rubio-Hurtado, Ruiz-Bueno \& Martínez-Olmo, 2016).

El rendimiento está relacionado con la tipología de estudiante. Hoy, el perfil del alumnado universitario es heterogéneo. Soler (2014) indica que solamente un $22 \%$ responde a un perfil tradicional, es decir, que su principal actividad sea el estudio. Es en este sentido que encontramos colectivos cuyas circunstancias no favorecen la persistencia en la carrera como los estudiantes que proceden de contextos socialmente desfavorecidos (Cabrera et al., 2012), los estudiantes que compaginan trabajo y estudio o los que proceden de ciclos formativos (Chen \& Carroll, 2007; Crawford \& Harris, 2008). 
Los estudiantes trabajadores son una realidad cada vez más visible en el contexto español (Finkel \& Barañaro, 2014). Estos autores señalan que el 46\% compagina los estudios con algún tipo de actividad remunerada y en el caso de ADE, los alumnos que compaginan ambas tareas alcanzan un $42 \%$. Los estudiantes que acceden a través de ciclos formativos también constituyen un colectivo con más probabilidades de abandonar (Figuera et al., 2015).

\section{Método}

\section{Objetivos}

El presente artículo, que describe el abandono de la carrera de ADE en primer, segundo y tercer curso, tiene por objeto: a) analizar el momento del abandono a lo largo del periodo de estudio; b) identificar las diferentes causas que hacen que el alumnado abandone; y c) describir los motivos de abandono a lo largo de los tres primeros cursos. La metodología utilizada ha sido de carácter cuantitativo descriptivo, utilizando un cuestionario como instrumento de recogida de información.

El trabajo se inscribe en el marco de dos proyectos $\mathrm{I}+\mathrm{D}$ realizados por el equipo TRALS de la Universidad de Barcelona (UB, en adelante). La persistencia y el abandono en el primer año de universidad en Ciencias Sociales: Bases para la mejora de la retención (EDU 2009-10351). Y trayectorias de abandono, persistencia y graduación en Ciencias Sociales: Validación de un modelo predictivo (EDU 2012-31563).

\section{Población y Muestra}

La población de referencia de los estudiantes de la cohorte 2010-11 de ADE, incluidos los de la doble titulación ADE-Derecho, que accede a la universidad es de 1.290. Los datos de la población y de la muestra (Tabla 1), han sido recogidos a partir de la base de datos institucional facilitada por la UB. El error muestral del conjunto de participantes es de 2.41 .

Tabla 1

Distribución de la población y muestra de abandono de la cohorte 2010-11 de ADE

\begin{tabular}{ccccccc}
\hline Población & $\begin{array}{c}\text { Población } \\
\text { abandono } \\
\text { 1er curso }\end{array}$ & $\begin{array}{c}\text { Muestra } \\
\text { abandono } \\
\text { 1er curso }\end{array}$ & $\begin{array}{c}\text { Población } \\
\text { abandono } \\
\mathbf{2}^{\mathbf{0}} \text { curso }\end{array}$ & $\begin{array}{c}\text { Muestra } \\
\text { abandono } \\
\mathbf{2}^{\mathbf{0}} \text { curso }\end{array}$ & $\begin{array}{c}\text { Población } \\
\text { abandono } \\
\text { 3er curso }\end{array}$ & $\begin{array}{c}\text { Muestra } \\
\text { abandono } \\
\text { 3er curso }\end{array}$ \\
\hline 1290 & 218 & 144 & 100 & 50 & 42 & 35 \\
\hline
\end{tabular}

En relación con los datos sociodemográficos de la muestra, el 59.6\% de las personas que abandonan son hombres y el $40.4 \%$ mujeres, su edad media oscila entre los 18 y 
21 años y el $42.7 \%$ trabaja. Un $82.7 \%$ han escogido ADE en primera opción. Un 45\% accede a la carrera a través del bachillerado, el 35.6\% a través de ciclos formativos, el $13.8 \%$ de otras carreras universitarias y el $6.2 \%$ como mayores de 25 años.

\section{Instrumento}

Para la recogida de los datos se ha utilizado un cuestionario telefónico de preguntas abiertas y cerradas, adaptado de la Entrevista telefónica de los alumnos que han abandonado de Torrado (2012), cuyas dimensiones son: estatus actual (estudia, trabaja, estudia y trabaja, inactivo), proceso y causas del abandono (momento de la decisión y causa principal del abandono), valoración académica y la situación académica actual. Se analizan las dos primeras. Respecto a la validez de contenido, de las causas del abandono se revisó la literatura especializada asegurando así la adecuación de la selección y representación de las variables. Y se utilizó la estrategia de juicios de expertos en educación.

\section{Procedimiento de recogida y análisis de datos}

El mes de enero del año posterior al curso académico estudiado, se realizan las llamadas del curso anterior asegurando, de este modo, la fiabilidad de los datos de la base de datos de la universidad. El análisis de los datos cuantitativos se realizó mediante el programa informático SPSS-Win.

La pregunta abierta sobre los motivos del abandono ha sido categorizada a partir del análisis de los ítems para confirmar su agrupación teórica en grandes factores explicativos del fenómeno y la revisión bibliográfica, para validar el constructo:

a) Roles compartidos (compaginación de la vida académica y laboral);

b) Variables personales (familia, salud, economía);

c) Variables psicológicas (desmotivación);

d) Estrategias de estudio (organización del estudio y resultados académicos);

e) Variables contextuales (integración y plan de carrera).

Asimismo, se ha realizado un análisis sobre los motivos del abandono a través del programa Iramuteq desarrollado por el Laboratoire d'Études et de Recherches Appliquées en Sciences Sociales del' Université de Toulouse. Este software permite un análisis multidimensional de textos y cuestionarios a partir del análisis estadístico del vocabulario del texto examinado (Lebart \& Salem, 1994). Proporciona varios tipos de análisis de los cuales se han utilizado el análisis de similitudes y el índice de especificidad. El primero remite a una técnica que se apoya en la teoría de los grafos para estudiar la proximidad y las relaciones entre los elementos de un conjunto y llegar a un grafo conexo, en este caso bajo la forma de un árbol (Degenne \& Vergès, 1973). El segundo, permite determinar qué elementos son característicos de un texto al realizar un estudio comparativo con otros textos a través del cálculo hipergeométrico. Así, surgen las palabras que aparecen en los textos de forma reiterada e infrautilizada. 


\section{Resultados}

\section{Análisis cuantitativo de los motivos del abandono}

El mayor número de abandono se produce antes de acabar el primer de año de estudios. Este porcentaje va disminuyendo curso a curso [F(2.20)=204.36; $p<.005]$ (HSD Tukey, Scheffe y Duncan).

Existen diferencias significativas en cuanto a los motivos de abandono $[\mathrm{F}(3.20)=10.25$; $p<.005]$. Mayoritariamente quien abandona continua estudios o compagina su formación con el trabajo y en menor medida se encuentra inactivo o sólo trabajando. La causa del abandono de los estudiantes que actualmente están inactivos estriba en las variables personales (cuestiones de salud, económicas o familiares). En cambio, aquellos que trabajan y estudian, la incompatibilidad de los roles es el detonante para decantarse por el trabajo. Los que abandonan, pero siguen estudiando, las variables psicológicas (desmotivación hacia los estudios o descontento con la formación) son el detonante para tomar la decisión.

La variable tipo de abandono se ha relacionado con los motivos de abandono. La prueba HSD Tukey y Duncan $[\mathrm{F}(4.30)=9.01 ; p<.005]$ expresa que independientemente de volver a estudiar o no las variables personales y los roles compartidos son las causas principales del abandono de los estudios de ADE. Esta realidad es independiente del año de abandono.

La Tabla 2 presenta los resultados del tipo de abandono, el estatus actual, el momento y su motivo.

\section{Tabla 2}

Tipo de abandono, estatus actual, momento de decisión y motivo del abandono por parte del estudiante según curso

\begin{tabular}{|c|c|c|c|c|c|c|c|c|c|c|c|}
\hline & \multicolumn{4}{|c|}{1 curso } & \multicolumn{4}{|c|}{2 curso } & \multicolumn{3}{|c|}{ 3r curso } \\
\hline $\begin{array}{l}\text { Tipo de } \\
\text { abandono }\end{array}$ & $\begin{array}{l}\text { REINC } \\
\mathrm{n}=100\end{array}$ & $\begin{array}{l}\mathrm{ESO} \\
4 \%\end{array}$ & $\begin{array}{c}\text { DESEI } \\
n=44\end{array}$ & $\begin{array}{l}\text { IÓN } \\
6 \%\end{array}$ & $\begin{array}{l}\text { REIN } \\
\mathrm{n}=40 \\
80 \%\end{array}$ & ESO & $\begin{array}{l}\text { DESER } \\
n=10 \\
20 \%\end{array}$ & IÓN & $\begin{array}{l}\text { REIN( } \\
n=26 \\
74.3 \%\end{array}$ & ESO & $\begin{array}{l}\text { DESER- } \\
\text { CIÓN } \\
\mathrm{n}=9 \\
25.75 \%\end{array}$ \\
\hline $\begin{array}{l}\text { Estatus } \\
\text { actual }\end{array}$ & $\begin{array}{l}\text { Est. } \\
\qquad \begin{array}{l}\mathrm{n}=51 \\
35.4 \%\end{array}\end{array}$ & $\begin{array}{l}\text { Est. } \\
\text { Tra- } \\
\text { baja } \\
n=49 \\
34.1 \%\end{array}$ & $\begin{array}{l}\text { Trab. } \\
n=33 \\
22.9 \%\end{array}$ & $\begin{array}{l}\begin{array}{l}\text { Inac- } \\
\text { tivo }\end{array} \\
n=11 \\
7.6 \%\end{array}$ & $\begin{array}{l}\text { Est. } \\
n=30 \\
60 \%\end{array}$ & $\begin{array}{l}\text { Est. } \\
\text { Tra- } \\
\text { baja } \\
n=10 \\
19.6 \%\end{array}$ & $\begin{array}{l}\text { Trab. } \\
n=8 \\
16.3 \%\end{array}$ & $\begin{array}{l}\text { Inac- } \\
\text { tivo } \\
\mathrm{n}=2 \\
4.1 \%\end{array}$ & $\begin{array}{l}\text { Est. } \\
n=14 \\
40 \%\end{array}$ & $\begin{array}{l}\text { Est. } \\
\text { Trab. } \\
\mathrm{n}=12 \\
34.3 \%\end{array}$ & $\begin{array}{l}\text { Trabaja } \\
\mathrm{n}=9 \\
25.7 \%\end{array}$ \\
\hline 2 meses & $\begin{array}{l}12 \\
23.5 \%\end{array}$ & $\begin{array}{l}15 \\
30.6 \%\end{array}$ & $\begin{array}{l}6 \\
18.2 \%\end{array}$ & & $\begin{array}{l}3 \\
10 \%\end{array}$ & & & & & & \\
\hline $\begin{array}{l}1 \text { semes- } \\
\text { tre }\end{array}$ & $\begin{array}{l}15 \\
29.4 \%\end{array}$ & $\begin{array}{l}16 \\
32.6 \%\end{array}$ & $\begin{array}{l}10 \\
30.3 \%\end{array}$ & $\begin{array}{l}8 \\
72.7 \%\end{array}$ & $\begin{array}{l}2 \\
6.7 \%\end{array}$ & $\begin{array}{l}1 \\
10 \%\end{array}$ & $\begin{array}{l}1 \\
12.5 \%\end{array}$ & & & & \\
\hline $\begin{array}{l}2 \text { semes- } \\
\text { tre }\end{array}$ & $\begin{array}{l}12 \\
23.5 \%\end{array}$ & $\begin{array}{l}6 \\
12.3 \%\end{array}$ & $\begin{array}{l}7 \\
21.2 \%\end{array}$ & $\begin{array}{l}1 \\
9.18 \%\end{array}$ & $\begin{array}{l}1 \\
3.3 \%\end{array}$ & $\begin{array}{l}4 \\
40 \%\end{array}$ & $\begin{array}{l}1 \\
12.5 \%\end{array}$ & & & $\begin{array}{l}1 \\
8.3 \%\end{array}$ & \\
\hline
\end{tabular}




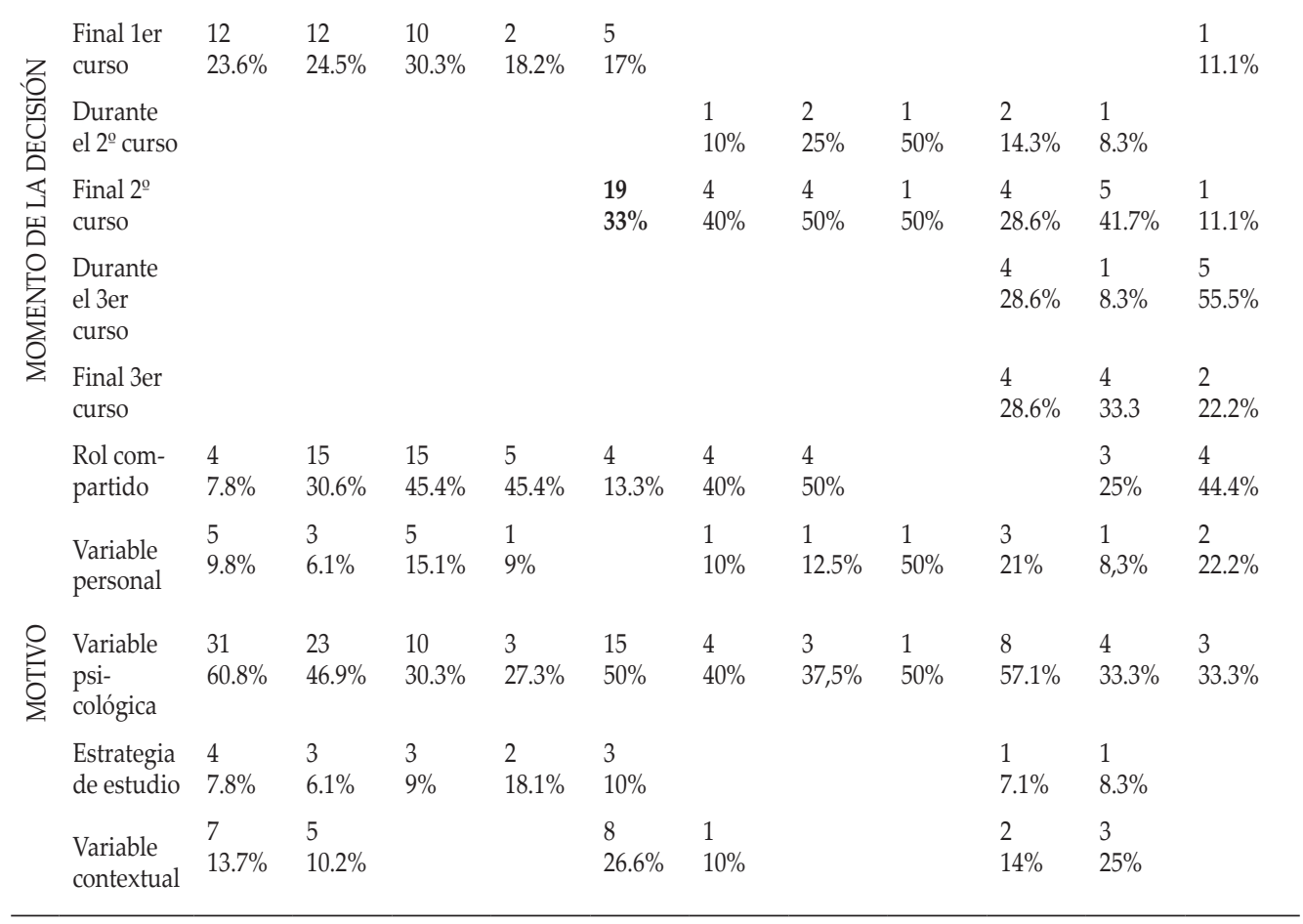

\section{Primer curso}

Podemos atribuir el abandono de los estudiantes de primero de ADE (que reingresa en el sistema educativo y que únicamente estudia en el momento de realizar la entrevista) a variables de naturaleza psicológica (al 60.8\% no le motiva la carrera). La decisión de abandonar no estuvo vinculada a ningún momento del curso académico en particular. La Tabla 2, muestra cómo los porcentajes se reparten entre los cuatro momentos del abandono.

Entre el estudiantado que reingresa al sistema educativo, y que compagina trabajo y estudios, también encontramos variables psicológicas en el motivo de abandono (no les satisfacía el grado al 46.9\%). Esta decisión se tomó, en la mayoría de los casos, durante el primer semestre (63.2\%): entre los dos primeros meses (30.6\%) y al finalizar el primer semestre (32.6\%). Por tanto, los estudiantes que reingresan abandonan ADE por motivos psicológicos, especialmente por ausencia de motivación hacia la carrera.

Para los estudiantes que no reingresan en el sistema educativo, concretamente los que en el momento de la entrevista únicamente trabajaban, la incompatibilidad entre estudio y empleo deviene el motivo principal de abandono (45.4\%). Una parte del estudiantado tomó la decisión al finalizar el primer semestre (30.3\%), y la otra, al concluir el curso (30.3\%). Los estudiantes inactivos decidieron abandonar a partir de finales del primer semestre $(72.7 \%)$ motivados por la imposibilidad de conciliar vida académica y laboral (45.4\%). 
En suma, los estudiantes que desertan del sistema educativo, tanto los que trabajan como los inactivos, abandonaron ADE por la imposibilidad de compaginar los roles de estudiante y trabajador.

\section{Segundo curso}

El alumnado que reingresa en el sistema educativo y que solo se dedica a los estudios, decide abandonar al final de segundo (33.3\%), a diferencia de aquellos que, estudiando y trabajando al mismo tiempo, lo hicieron en dos momentos distintos: segundo semestre (40\%) y final del segundo curso (40\%). Señálese que ambos grupos de estudiantes van arrastrando la decisión de abandono desde primero.

Aquí, el motivo principal de abandono también está sujeto a cuestiones psicológicas: desmotivación y descontento con la carrera. Asimismo, cabe destacar los estudiantes (40\%) que, tratando de compaginar estudio y empleo, deciden abandonar por las dificultades de compatibilizar ambas tareas.

En relación con los estudiantes de "deserción" que únicamente trabajaban en el momento de la entrevista, señalamos que, como en primero, la incompatibilidad entre el estudio y el trabajo vuelve a ser el motivo de abandono (50\%), seguido de las variables psicológicas (37.5\%). La mayoría de ellos ha tomado la decisión de abandonar en segundo.

\section{Tercer curso}

Los datos indican que el alumnado que llega a tercero y abandona, inició la carrera con determinación y la mantiene hasta la mitad del segundo curso. A partir de este momento, se suceden una serie de motivos, vinculados a variables psicológicas, que les conminan a abandonar la carrera aunque continúan estudiando. Este proceso es unitario tanto para el estudiantado que trabaja y estudia, como para el que únicamente estudia.

En el caso de los estudiantes que desertan, cabe destacar que todos trabajaban en el momento de la entrevista y que abandonaron, en su mayoría, durante el tercer curso $(77.7 \%)$. La decisión viene tomada por las dificultades de compaginación entre la esfera laboral y académica, y por motivos psicológicos como la desmotivación o el descontento.

\section{Análisis lexicométrico de los motivos del abandono}

La Tabla 3 recoge los diferentes elementos que componen los corpus del relato para cada uno de los cursos. Confirma un comportamiento común en los trabajos de análisis lexicométrico: en los hápax, alrededor del 70\% del total son formas (palabras que no se repiten) y las ocurrencias (palabras que se repiten en diferentes frecuencias) alrededor del $30 \%$. Así sucede en segundo y tercer curso, en los que el porcentaje de las ocurrencias se acerca al 30\%, pero no en primero puesto que los motivos de abandono son mucho más diversos. Así, el discurso de estos estudiantes se compone de menor número de palabras que se repiten en diferentes frecuencias. 
Tabla 3

Estadísticas generales de los corpus textuales del abandono de los tres cursos

\begin{tabular}{lccc}
\hline & Abandono de 1r & Abandono de 2n & Abandono de 3r \\
\hline Textos & 1 & 1 & 1 \\
Segmentos & 71 & 18 & 35 \\
Ocurrencias & 2589 & 620 & 1266 \\
Formas & 638 & 250 & 453 \\
Hapax & $39561 \%$ formas & $16766.8 \%$ formas & $30567.33 \%$ formas \\
& $15.26 \%$ ocurrencias & $24.94 \%$ ocurrencias & $24.09 \%$ ocurrencias \\
\hline
\end{tabular}

\section{Primer curso}

Como puede apreciarse en la Figura 1, el principal núcleo de ocurrencias se estructura alrededor de la palabra GUSTAR que se relaciona, a su vez, con otros tres: 1. CARRERA, TRABAJAR, IR y CLASE; 2. ESTUDIO, TRABAJO y COMPAGINAR 3. QUERER.

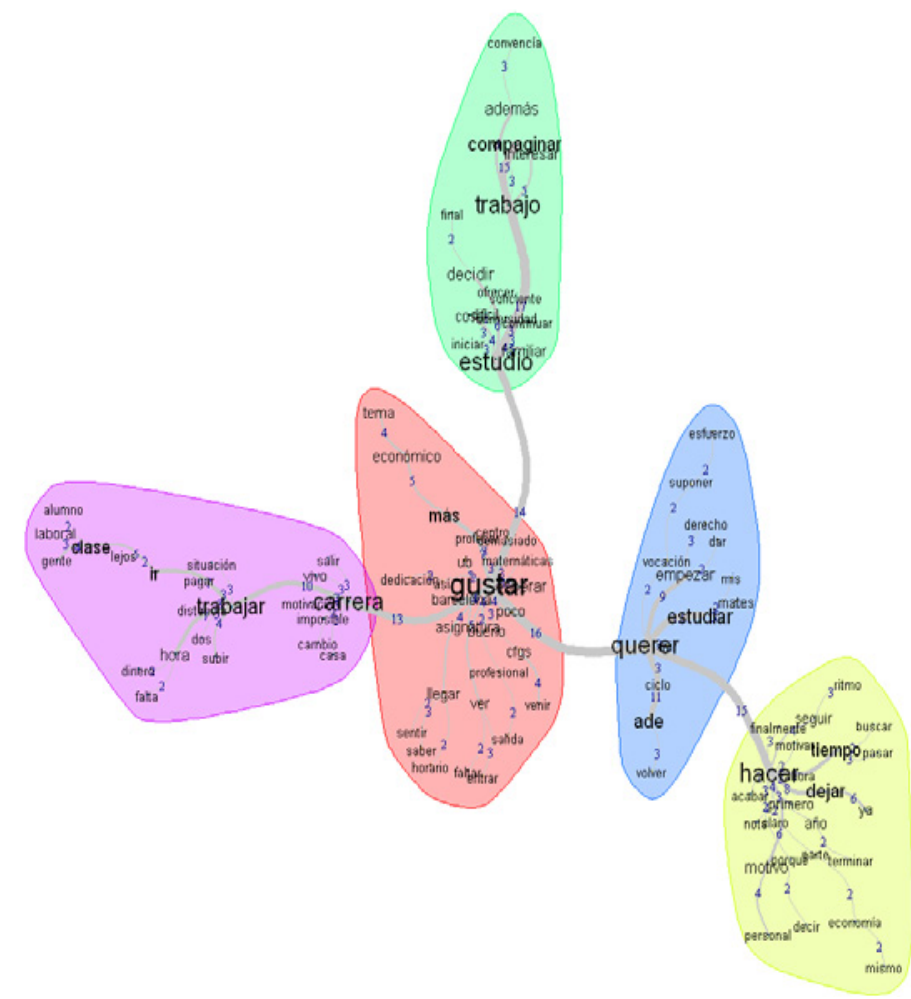

Figura 1. Árbol del análisis de similitud de los motivos de abandono de primer curso. 
La recurrencia con la que los estudiantes utilizan la palabra GUSTAR en su discurso, nos remite a distintos motivos de abandono para primero. No les gusta la carrera porque: no es lo que QUERIAN estudiar/hacer en un inicio; por la dificultad para compaginar estudios y trabajo y, porque el TRABAJO obstaculiza la asistencia a clase.

El motivo principal de abandono en el primer curso de ADE se recoge en la frase "No me gusta" que está relacionada con dos elementos. Por un lado, se vincula con el imaginario de los estudiantes sobre la universidad desconociendo tanto las formas de trabajo universitario, como la cultura de la institución. Así, estos estudiantes construyen unas expectativas previas al comienzo de la carrera que se ven defraudadas durante los primeros meses. De ahí la recurrencia de la frase "No era lo que me esperaba".

Por otro lado, el rendimiento académico difiere según las diversas vías de acceso a la universidad. Los estudiantes que acceden a ADE a través de ciclos formativos señalan que carecen del nivel académico necesario para sostener las exigencias de estudio de la carrera y terminan abandonando. Tal como indican diversas investigaciones (Figuera \& Torrado, 2013; Gairín et al. 2010; MECD, 2015; Torrado, 2012).

Soler (2014) señala que alrededor de la mitad del alumnado presenta actitudes periféricas, esto es, una vinculación débil con los estudios. En este sentido, la significación que construyen en torno al ser estudiante universitario está desprovista de la necesidad de tener que dedicar un esfuerzo y tiempo prolongado al estudio, como sucede con la asignatura de matemáticas. Estas actitudes periféricas, como muestran los siguientes núcleos, también son atribuidas al alumnado que trata de compaginar la esfera laboral con la académica (Soler, 2014).

En el núcleo CARRERA, TRABAJO y CLASE, la palabra principal es Carrera. Aquí se solapan dos cuestiones que intervienen en la decisión de abandonar. Primera, a los estudiantes no les gusta la carrera, hecho al que se le añade la dificultad para compaginar el trabajo con la asistencia a las clases. Segunda, podría realizarse una interpretación inversa. A saber, el hecho de no poder ir regularmente a clase les aleja paulatinamente de la universidad, llegando a la conclusión de que no les gusta la carrera.

El núcleo COMPAGINAR, ESTUDIO Y TRABAJO aparece vinculado al alumnado cuya actividad primordial es la laboral. El principal motivo de abandono es la imposibilidad de compaginar estudio y trabajo tal como sucede en otras investigaciones (Chen \& Carroll, 2007; Crawford \& Harris, 2008; Finkel \& Barañaro, 2014) que sitúan a los estudiantes trabajadores como un colectivo vulnerable y en riesgo. Si bien muchos de éstos dedican más tiempo al estudio que aquellos que no trabajan, los resultados académicos no siempre muestran este esfuerzo, aspecto que viene a reforzar los motivos de abandono. Otros buscan alternativas que les permitan compaginar de un modo más óptimo el estudio con el trabajo y, así, continuar con la carrera. En ese sentido, los que abandonan los estudios en la UB se matriculan en universidades con modalidades no presenciales.

Las palabras del núcleo QUERER y ESTUDIAR adquieren significado en el marco de la elección de la carrera. Una elección que, podría decirse, se realiza sin demasiada convicción y que encontramos resumida en otra de las frases recurrentes entre el alumnado: "No era lo que quería estudiar"; elección basada en lo que Navarro y 
Soler (2014, p. 64) llaman "motivación expresiva" en alusión al "interés específico por la disciplina que se elige y se estudia". Los estudiantes de este núcleo abandonan ADE, precisamente, porque su motivación expresiva está puesta en otro tipo de carreras.

El análisis del núcleo HACER es similar al anterior. El verbo hacer se utiliza coloquialmente como sustituto de un sinfín de acciones. En ese caso, el verbo ESTUDIAR se sustituye por HACER. La frase que viene a sintetizar esta cuestión es: "No me gustaba y por lo tanto, no era lo que quería hacer".

\section{Segundo curso}

La dimensión discursiva del alumnado de segundo año se centra en la palabra CARRERA. Como puede observarse en la Figura 2, surgen tres ramas de este núcleo: GUSTAR, ESTUDIO y una tercera, que no termina de concretarse en ninguna palabra específica y que, por tanto, no será objeto de análisis. A partir de este árbol, se podría decir, que la carrera tiene una carga de matemáticas excesiva para muchos estudiantes; la carrera continúa sin terminar de gustarles y, finalmente, existen dificultades de compaginación entre estudio y trabajo.

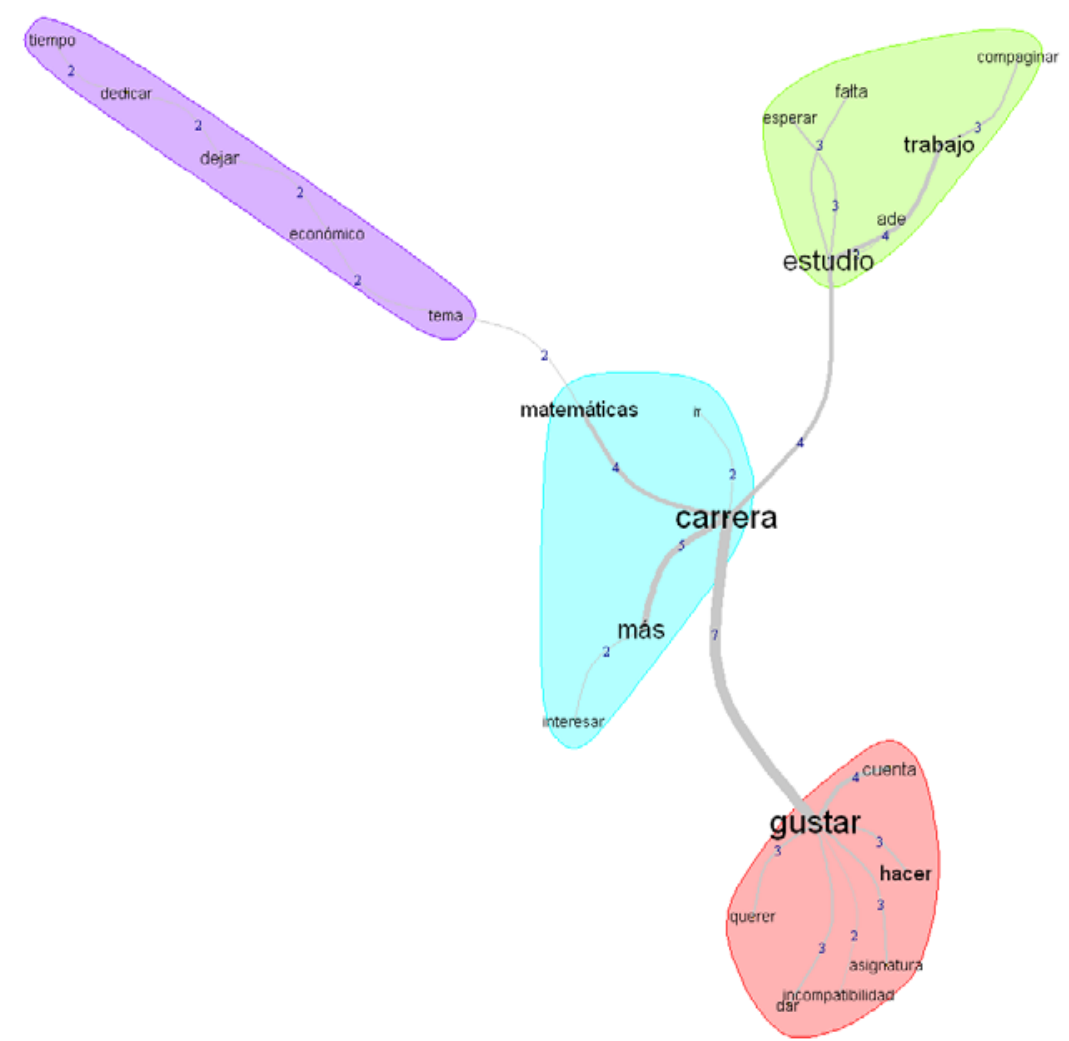

Figura 2. Árbol del análisis de similitud de los motivos de abandono de segundo curso. 
El núcleo central de esta arborescencia gira alrededor de la palabra CARRERA que, siguiendo el discurso de los estudiantes, nos remite a la idea de una carrera con un grado de dificultad elevado, especialmente por lo referente a las materias de matemáticas y estadística. A pesar de los cursos preparatorios que ofrece la Facultad de Economía y Empresa para reforzar los aprendizajes en dichas materias, los estudiantes continúan teniendo serias dificultades. Los obstáculos con las matemáticas suponen un motivo no menor de abandono.

Si la motivación expresiva hacia la carrera aumenta en el segundo curso (Navarro \& Soler, 2014), podría sugerirse que la desmotivación expresiva también crece entre aquellos estudiantes que no han tomado todavía la decisión de abandonar. Es lo que surge en el núcleo GUSTAR. Álvarez et al. (2015) sitúan que un porcentaje importante del alumnado no tienen claro el qué y el para qué de lo que estudian. El desconocimiento sobre la formación puede incentivar la desmotivación entre los estudiantes y abrir una puerta hacia el abandono de la carrera.

Finalmente, la palabra ESTUDIO, nos remite a las dificultades de los estudiantes para compaginar vida académica y laboral. De nuevo, el colectivo de alumnos que tiene un empleo deviene un grupo de riesgo (Chen \& Carroll, 2007; Crawford \& Harris, 2008). Al mismo tiempo, y como sucede en primero, encontramos estudiantes que, además de las dificultades para conciliar estudio y empleo, sitúan que la carrera no cumple con sus expectativas.

\section{Tercer curso}

El discurso de los estudiantes se centra en el núcleo ESTUDIO que, como puede apreciarse en la Figura 3, contiene, a su vez, un pequeño núcleo con las palabras año, primero y segundo. ESTUDIO se relaciona con el núcleo GUSTAR, de la que surgen dos ramificaciones: una formada por la palabra QUERER y otra formada por TITULACIÓN y DERECHO. Encontramos un último núcleo alrededor de la palabra HACER.

En este árbol se observa la desmotivación hacia el ESTUDIO que lleva a los estudiantes a expresar que no volverían a HACER ADE. Han sostenido la carrera por una motivación profesional que, en tercero, se desmorona. Así, los verbos GUSTAR y QUERER continúan conjugándose en negativo en relación ya no con la carrera sino a la propia profesión.

Cuando los estudiantes de tercero relatan sus motivos de abandono alrededor de la palabra ESTUDIO, refieren situaciones ya detectadas durante el primer curso pero que, ahora, aparecen de una forma más acentuada. De ahí que este núcleo aparezca acompañado de un sub-núcleo con las palabras: año, primero y segundo. Si bien, en los cursos anteriores, una de las causas relevantes de abandono era la incompatibilidad entre universidad y trabajo, en tercero, encontramos la desmotivación como motivo principal que viene arrastrándose desde los años previos.

Los alumnos de tercero conjugan el verbo GUSTAR como en los cursos anteriores: "No me gusta". A estas alturas de la carrera, podemos indicar que uno de los motivos de esa insatisfacción subyace en los escollos académicos que han encontrado con las materias de contenido matemático. Esta cuestión se ve agravada, en ocasiones, con las dificultades recurrentes para compaginar trabajo y estudio. Ambos factores terminan desencadenando el abandono. 
Es preciso señalar que este grupo de estudiantes, a pesar de su desmotivación, sostiene la carrera hasta el tercer curso gracias a una cierta motivación profesional. Aun así, no es suficiente. Terminan abandonando el grado por los obstáculos que le atribuyen al plan de estudios. No son pocos los estudiantes que, como señalábamos con anterioridad, desconocen el qué y para qué de lo que estudian (Álvarez et al., 2015). Esta cuestión aparece como uno de los factores de abandono a considerar.

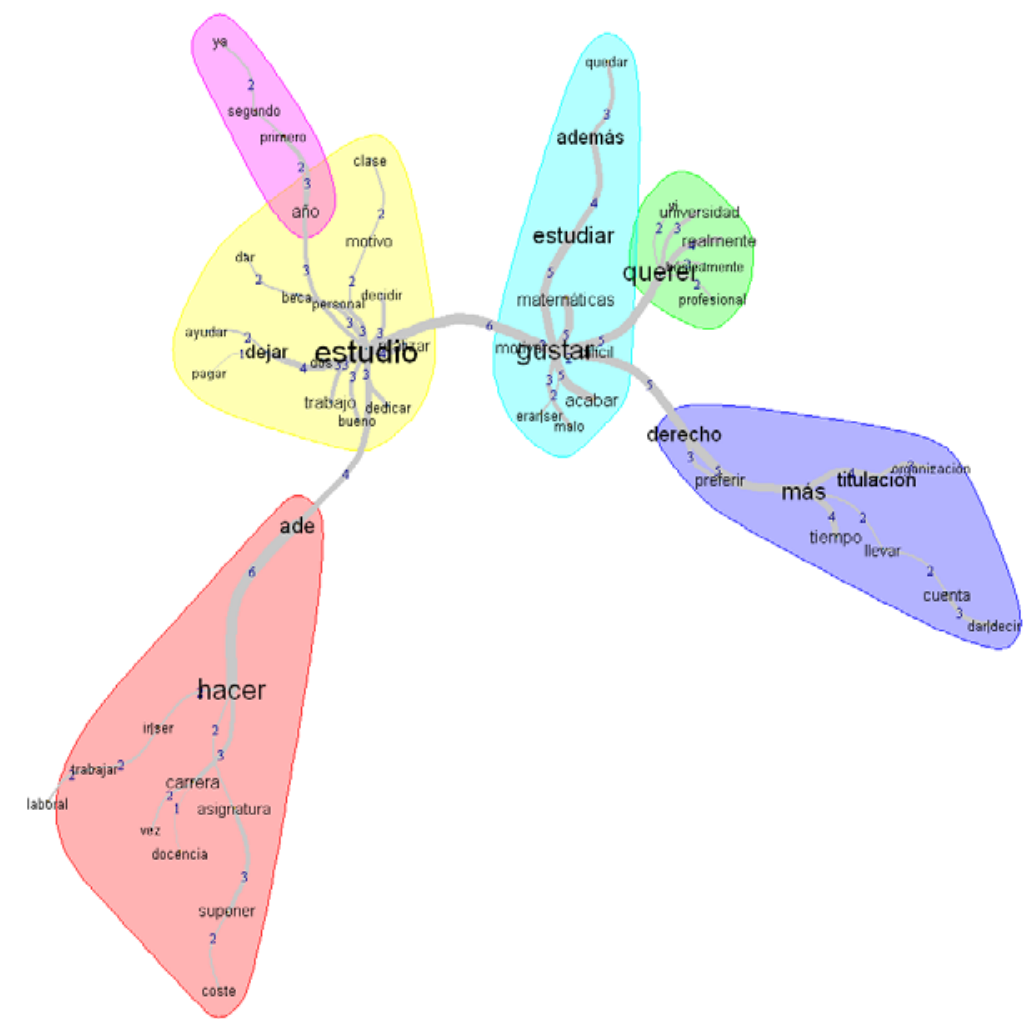

Figura 3. Árbol del análisis de similitud de los motivos de abandono de tercer curso.

Entre el alumnado de tercero, el verbo QUERER, ya no se refiere tanto a "no querer" estudiar la carrera, como a no desear el futuro profesional que les depara ADE. En este curso, se detecta una cierta desmotivación en relación con la dimensión profesional de la carrera.

El núcleo DERECHO, TITULACIÓN está formado, principalmente, por las respuestas de los estudiantes de la doble titulación. Ante las dificultades que comporta la realización de un doble grado, los estudiantes se ven en la disyuntiva de elegir una u otra carrera y se decantan por Derecho. La falta de motivación deviene, una vez más, un factor clave de abandono. En este sentido, los alumnos manifiestan descontento con relación a la organización académica de la doble titulación que les dificulta ostensiblemente el tiempo para estudiar. 


\section{Especificidades}

La Figura 4 muestra las palabras más utilizadas y más infrarrepresentadas por los estudiantes que dan significado al abandono en los tres cursos con especificidades distintas (por encima de 0 , positivo; por debajo del 0 , negativo).

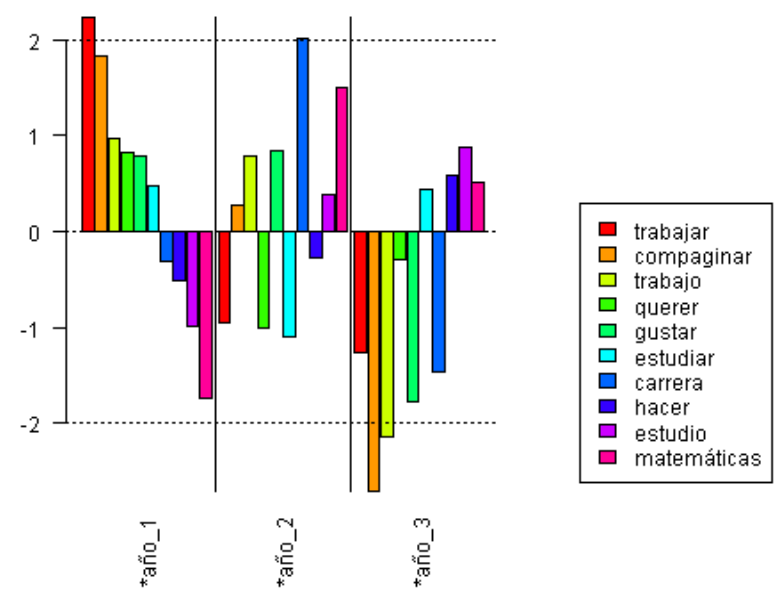

Figura 4. Índice de especificidades de las palabras de los distintos cursos.

Las más representadas en primero trabajar, compaginar, trabajo, querer y gustar se infrautilizan en tercero.

La triada COMPAGINAR-TRABAJAR Y TRABAJO remite a la compartimentación de los roles empleo/carrera a la que se ven abocados los estudiantes. Durante el primer curso, uno de los motivos reiterados de abandono es la imposibilidad de compaginar ambos roles. En segundo, esta dificultad es nombrada en menor medida. ¿Qué podría indicar? ¿Qué los estudiantes que continúan trabajando han encontrado los recursos para la conciliación de ambas esferas? ¿Qué quedan muy pocos estudiantes trabajadores? El segundo curso parece ser el decisivo para abandonar por motivos laborales puesto que, en tercero, estas palabras se presentan bajo una especificidad negativa. De este modo, pasado el ecuador de la carrera, o bien los estudiantes no trabajan, o el trabajo ya no les resulta un obstáculo para continuar los estudios.

El verbo QUERER solamente cuenta con especificidad positiva en primero. Y es que la elección de los estudios tiene sus efectos en ese momento. Son los estudiantes que no querían estudiar ADE y por distintas circunstancias se vieron impelidos a matricular este grado.

La especificidad de Gustar es positiva en los dos primeros cursos. Los estudiantes abandonan porque no les gusta ADE. Es significativo que en segundo se continúe esgrimiendo este motivo. Podría aventurarse que los estudiantes se dan un curso académico más para comprobar que, en efecto, no les gusta la carrera.

En el segundo curso, Carrera y Matemáticas son las palabras más empleadas. En este curso, las matemáticas aparecen como una fuente incontestable de obstáculos. En 
primero, los estudiantes empiezan a vislumbrar su dificultad, pero apenas la nombran. En segundo, se hace evidente el problema, puesto que son las asignaturas que los estudiantes repiten y el problema continúa en tercero, pero con menor intensidad.

En tercero, las palabras Estudio y Hacer adquieren importancia. Ahora, las particularidades del abandono se anclan en la experiencia acumulada de estos años en la universidad. Ya no es "No quiero HACER ADE" como en primer curso, sino que se convierte en "No volvería a HACER ADE". Han tenido que pasar tres años para tomar esta decisión.

\section{Discusión y conclusiones}

Los resultados confirman la línea actual del abandono. Éste se produce en ADE mayoritariamente en el primer curso y toman la decisión durante el primer trimestre, tal como señala la literatura. El abandono universitario no debe interpretarse únicamente como el abandono del sistema educativo. Un número significativo de estudiantes reingresa en otros grados universitarios o ciclos formativos. Esto sucede en los tres cursos analizados, registrándose en primero el menor porcentaje. Es en este curso donde se producen las mayores cifras de abandono. Esta realidad nos lleva a cuestionar la calidad de la orientación antes de la entrada a la universidad puesto que el relato de estos estudiantes de primero se estructura alrededor de la frase: “No me gusta". Podría ser una elección inadecuada de la carrera (Corominas, 2001; Navarro \& Soler, 2014). De ahí la importancia de la orientación (Álvarez, 2015; Romero \& Figuera, 2016). Si bien ésta está más arraigada en la elección de la carrera, tiene que ampliarse hacia la reflexión de la trayectoria personal del estudiante y sus expectativas de futuro. Ello puede permitir al estudiante visualizar posibles obstáculos como la compaginación de roles de estudiante y trabajador, como sucede frecuentemente en el alumnado proveniente de ciclos formativos y mayores de 25 años.

Las causas del abandono para los estudiantes que reingresan son diferentes según su estatus. Por un lado, en los tres cursos, aquellos que solamente estudian dejan la carrera por desmotivación. El análisis lexicométrico permite ahondar en esta desmotivación. El abandono de primer curso se estructura alrededor de "No quería estudiar ADE" y de "No me gusta". Después de un primer semestre en la universidad, el estudiantado que abandona se da cuenta que realmente no es lo que esperaba. ¿Qué esperaba de la Universidad? ¿De las asignaturas? ¿Del ambiente? ¿Del profesorado? En segundo, este gustar se concreta y surge la oración "No me gusta la carrera". Es una carrera difícil con muchas matemáticas. En tercero, algunos estudiantes siguen sintiéndose insatisfechos con los estudios. La construcción de su futura identidad profesional no les atrae. Por otro lado, un número importante de estudiantes que compaginan trabajo y estudios se ven obligados a abandonar ante la incompatibilidad de ambas esferas. No obstante, una parte reingresa en universidades virtuales para continuar el mismo grado. Como indica el análisis lexicométrico, esto es especialmente notorio en el primer curso, que se reduce en segundo. Los estudiantes que trabajan y estudian ya no llegan a tercero o han buscado alguna solución para la conciliación.

En la línea de lo anteriormente planteado, cabe subrayar el significado que los estudiantes le otorgan al hecho de simultanear esfera académica y laboral. Los resultados 
indican que su actividad laboral impacta y condiciona la continuidad de los estudios. Esos estudiantes se confrontan a lo largo del primer curso con los obstáculos propios de simultanear ambas actividades, pero no es hasta segundo que toman la decisión de abandonar. Estos elementos abren un interrogante en relación con las actuales políticas de permanencia. Si un número creciente de estudiantes se ve abocado a simultanear trabajo y estudio, tal vez sea pertinente sugerir un replanteamiento en los itinerarios formativos universitarios basados, preeminentemente, en la presencialidad. La equidad en la inclusión en la enseñanza superior queda asimismo en entredicho si observamos que, como muestra la investigación, la deserción entre los grupos no convencionales es más notorio que en el resto.

Otros de las cuestiones ligadas a ADE es la exigencia en las materias de matemáticas. El constante fracaso académico lleva a los estudiantes a abandonar en cursos posteriores a primero. Como expresan Navarro y Soler (2014, p. 71), el incremento de la motivación expresiva en los cursos posteriores se explicaría por el siguiente hecho: "el éxito académico suele convertirse en el principal elemento motivador intrínseco del propio avance en los estudios". En este sentido, ciertas materias de la titulación podrían ser predictivas del futuro abandono, dato que podría ayudar a los responsables de la titulación a crear planes específicos de acción tutorial y apoyo académico que contribuyeran a prevenir dichas deserciones (Álvarez, 2013). Existe una estrecha vinculación entre las metodologías docentes y la (des)motivación del alumnado. Dichas metodologías influyen directamente en las actividades de aprendizaje, la motivación y el seguimiento de los estudios (Freixa, Aparicio \& Triadó, 2014; Mingorance \& Calvo, 2012; Rubio-Hurtado et al., 2016), de modo que podría sugerirse una relación de causalidad entre metodologías docentes inadecuadas y motivos de abandono. Nuestro estudio vislumbra esta conexión abriendo así una línea de investigación futura.

Finalmente, hay que considerar la construcción de la identidad profesional. Los estudiantes que, habiendo llegado a tercero, abandonan, lo hacen por una decepción en relación con el rol profesional que habían imaginado. En el transcurso de los tres años, van tomando consciencia entre el ideal de la profesión y la realidad. No se sienten atraídos por la identidad profesional asociada a la carrera. Por ello, cabría sugerir la pertinencia de espacios, especialmente en primero, que permitieran a los estudiantes comenzar a confrontar y construir una identidad profesional. Este aspecto es especialmente sensible para los estudiantes que acceden de ciclos formativos. Dicho desencanto es más notorio entre éstos puesto que cuentan con una visión profesional previa.

\section{Referencias}

Álvarez, M. (2015). La orientación y la tutoría universitaria en la formación de grados y postgrados. Las transiciones académicas. En P. Figuera (Coord.), Persistir con éxito en la universidad: De la investigación a la acción (pp. 175-204). Barcelona: Laertes.

Álvarez, P. (2013). La tutoría como eje articulador del proceso de aprendizaje del alumnado universitario. Revista Qurriculum, 26, 73-87.

Arce, M.E., Crespo, B., \& Miguez-Álvarez, C. (2015). Higher Education Drop-Out in Spain-Particular Case of Universities in Galicia. International Education Studies, $8(5), 247-264$. 
Álvarez, P., López, D., \& Pérez-Jorge, D. (2015). Análisis de los factores que intervienen en la transición del bachillerato a la universidad. En AIDIPE (Ed.), Investigar con y para la sociedad (pp. 599-608). Cádiz: Bubok. Recuperado de http://aidipe2015.aidipe.org

Beaupère, N., Chalumeau, L., Gury, N., \& Hugree, C. (2007). L'abandon des études supérieures. Rapport réalisé pour l'Observatoire national de la vie étudiante. París (Francia): La Documentation Française.

Berlanga, V., Figuera, P., \& Pons-Fanals, E. (2013). Incidencia de la beca salario: impacto, perfil y rendimiento de los estudiantes. RELIEVE, 19(2), 1-15. doi: 10.7203/ relieve.19.2.2654

Bethencourt, J.T., Cabrera, L. Hernández, J.A., Álvarez, P., \& González, M. (2008). Variables psicológicas y educativas en el abandono universitario. Revista electrónica de Investigación Psicoeducativa, 6(3), 603-622.

Braxton, J., Hirschy, A., \& McClendon, S. (2004). Understanding and reducing college students' departure. San Francisco (EEUU): Jossey-Bass.

Cabrera, A.F., Burkum, K.R., La Nasa, S.M., \& Bibo. E. (2012). Pathways to a four-year degree: Determinants of degree completion among socio-economically disadvantaged students. En A. Seidman (Ed.), College Student Retention: A Formula for Student Success (pp. 155-209). Westport, Conneticut (EEUU): Praeger Publishers.

Chen, X., \& Carroll, C. D. (2007). Part-time undergraduates in post-secondary education: 2003-04 (NCES 2007-165. U.S. Department of Education). Washington, DC (EEUU): National Center for Education Statistics.

Corominas, E. (2001). La transición de los estudios universitarios. Abandono o cambio en el primer año de universidad. Revista de Investigación Educativa, 19(1), 127-151.

Crawford, K., \& Harris, M. (2008). Differential predictors of persistence between community college adult and traditional aged students. Community College Journal of Research and Practice, 32, 75-100.

Degenne, A., \& Vergès, P. (1973). Introduction à l'analyse de similitude. Revue Francaise de Sociologie, 14(4), 471-512.

Escandell, O., Marrero, G., \& Rubio, F. (1999). El abandono de los estudiantes de la Universidad de Las Palmas de Gran Canaria. Revista Electrónica Interuniversitaria de Formación del Profesorado, 2(1) pp. 673-680. Recuperado de http://m.aufop.com/ aufop/uploaded_files/articulos/1224339869.pdf

Figuera, P., \& Torrado, P. (2013). El contexto académico como factor diferenciador en la transición a la universidad. Contrapontos, 13(1), 33-41.

Figuera, P., Torrado, M., Dorio, I., \& Freixa, M. (2015). Trayectorias de persistencia y abandono de estudiantes universitarios no convencionales: Implicaciones para la orientación. Revista Electrónica Interuniversitaria de Formación del Profesorado, 18(2), 107-123.

Finkel, L., \& Barañaro, M. (2014). La dedicación al estudio y al trabajo de los estudiantes universitarios en España. Revista de la Asociación de Sociología de la Educación, 7(1), 82-103.

Freixa, M., Aparicio, P., \& Triadó, X. (2014). El rol del profesorado como elemento clave en las Instituciones y en el contexto de la educación superior. En P. Figuera (Coord.), Persistir con éxito en la universidad: De la investigación a la acción (pp. 139157). Barcelona: Laertes. 
Gairín, J., Figuera, P., \& Triadó, X. (2010). L'abandonament dels estudiants a les universitats catalanes. Barcelona: AQU.

Krause, K., \& Coates, H. (2008). Students' engagement in first-year University. Assessment and Evaluation in Higher Education, 3(5), 493-505.

Lebart, L., \& Salem, A. (1994). Statistique textuelle. París (Francia): DUNOP.

Mingorance, C., \& Calvo, A. (2012). Los resultados de los estudiantes en un proceso de evaluación con metodologías distintas. Revista de Investigación Educativa, 31(1), 275-293.

Ministerio de Educación Cultura y Deporte [MECD] (2015). Datos y cifras del sistema universitario español. Curso 2015-2016. Madrid: Ministerio de Educación, Cultura y Deporte.

Navarro, P., \& Soler, I. (2014). Las motivaciones de la elección de carrera por los estudiantes universitarios. Revista de la Asociación de Sociología de la Educación, 7(1), 61-81.

Nora, A., \& Crips, G. (2012). Student persistence and degree attainment beyond the first-year in college: Existing knowledge and directions for future research. En A. Seidman (Ed.), College Student Retention (pp. 229-251). Westport, CT (EEUU): Rowan y Littlefield publishers.

Romero, S., \& Figuera, P. (2016). La orientación en la universidad. En A. Manzanares (Ed.), Orientación a lo largo de la vida (pp. 163-196). Madrid: Wolters Kluver.

Rubio-Hurtado, M.J., Ruiz-Bueno, A., \& Martínez-Olmo, F. (2016). Percepción del alumnado sobre la utilidad de las actividades de aprendizaje para desarrollar competencias. Revista de Investigación Educativa, 34(1), 221-240.

Soler, I. (2014). Una tipología de la población estudiantil universitaria. Revista de la Asociación de la Sociología de la Educación, 7(1), 104-122.

Torrado, M. (2012). El fenómeno del abandono en la Universidad de Barcelona: El caso de ciencias experimentales (Tesis doctoral, Universidad de Barcelona). Recuperado de http://www.tdx.cat/bitstream/handle/10803/134955/01.MTF_TESIS.pdf?sequence=9

Yorke, M., \& Longden, B. (2008). The first-year experience of higher education in the UK. York, UK: The Higher Education Academy.

Fecha de recepción: 30 de diciembre de 2016.

Fecha de revisión: 5 de mayo de 2017.

Fecha de aceptación: 11 de mayo de 2017. 